\title{
In-Channel Responsive Surface Wettability for Reversible and Multiform Emulsion Droplet Preparation and Applications
}

Lanhui Li, ${ }^{\dagger}, Z^{\dagger}$ Zhibin Yan, ${ }^{\dagger}$ Mingliang Jin, ${ }^{\dagger}$ Xiangshen You, ${ }^{\dagger}$ Shuting Xie, ${ }^{\dagger}$ Zhenping Liu, Albert van den Berg, Jan C. T. Eijkel, ${ }^{\ddagger}$ and Lingling Shui ${ }^{\ddagger}, \dagger, \S_{(1)}$

${ }^{\dagger}$ International Joint Laboratory of Optofluidic Technology and System (LOTS), National Center for International Research on Green Optoelectronics \& South China Academy of Advanced Optoelectronics, and ${ }^{\S}$ School of Information and Optoelectronic Science and Engineering, South China Normal University, Guangzhou 510006, China

${ }^{\ddagger}$ BIOS/Lab on a Chip Group, MESA+ Institute for Nanotechnology, University of Twente, Enschede 7500AE, The Netherlands

\section{Supporting Information}

ABSTRACT: We report on a simple approach for in-channel functionalization of a polydimethylsiloxane (PDMS) surface to obtain a switchable and reversible wettability change between hydrophilic and hydrophobic states. The thermally responsive polymer, poly(N-Isopropylacrylamide) (PNIPAAm), was grafted on the surface of PDMS channels by UV-induced surface grafting. PNIPAAm-grafted PDMS (PNIPAAm-g-PDMS) surface wettability can be thermally tuned to obtain water contact angles varying in the range of 24.3 to $106.1^{\circ}$ by varying temperature at $25-38$

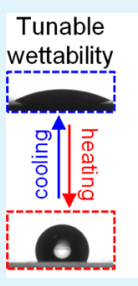

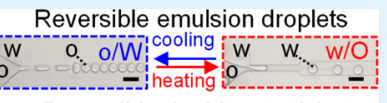

Reversible double emulsion
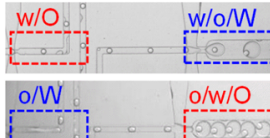
W/ON ${ }^{\circ} \mathrm{C}$. By selectively modifying the functionalized area in the microfluidic channels, multiform emulsion droplets of oil-in-water $(\mathrm{O} / \mathrm{W})$, water-in-oil $(\mathrm{W} / \mathrm{O})$, oil-in-water-in-oil (O/W/O), and water-in-oil-inwater $(\mathrm{W} / \mathrm{O} / \mathrm{W})$ could be created on-demand. Combining solid surface wettability and liquid-liquid interfacial properties, tunable generation of $\mathrm{O} / \mathrm{W}$ and $\mathrm{W} / \mathrm{O}$ droplet and stratified flows were enabled in the same microfluidic device with either different or the same two-phase fluidic systems, by properly heating/cooling thermal-responsive microfluidic channels and choosing suitable surfactants. Controllable creation of $\mathrm{O} / \mathrm{W} / \mathrm{O}$ and $\mathrm{W} / \mathrm{O} / \mathrm{W}$ droplets was also achieved in the same microfluidic device, by locally heating or cooling the droplet generation areas with integrated electric heaters to achieve opposite surface wettability. Hollow microcapsules were prepared using double emulsion droplets as templates in the microfluidic device with sequential hydrophobic and hydrophilic channel segments, demonstrating the strength of the proposed approach in practical applications.

KEYWORDS: surface wettability, thermoresponsive, microfluidics, droplet, reversible emulsion, multi-emulsion, microcapsule

\section{INTRODUCTION}

Wettability is one of the key factors related to functionalities in biological system, ${ }^{1}$ cell membrane remodeling, ${ }^{2}$ water transport in earth soil, ${ }^{3}$ wetting behavior of liquid on surfaces, ${ }^{4}$ protein and bimolecular adsorption, ${ }^{5}$ nanomaterial synthesis, ${ }^{6}$ and sustainability of photoelectric devices. ${ }^{7}$ Surface wettability is also a key factor in handling complex fluidic systems involving both polar and nonpolar fluids, such as emulsions. Emulsions composed of two or more immiscible liquids are used in a wide range of industries such as cosmetic, food, agriculture, and pharmaceutical industries. ${ }^{8}$ Emulsion types basically including $\mathrm{O} / \mathrm{W}, \mathrm{W} / \mathrm{O}, \mathrm{O} / \mathrm{W} / \mathrm{O}$, and $\mathrm{W} / \mathrm{O} / \mathrm{W}$ emulsions have been well developed for preparing stable micro-/nano-droplets or microcapsules, which are applied as food emulsion, or for topical applications such as for diagnostics and drug delivery. ${ }^{9,10}$ However, for some special applications such as butter making and for applications in the petrochemical industry, emulsion reversion is a necessity that still is in need of more study. ${ }^{8,11,12}$

Droplet-based microfluidics has emerged as a popular technology to generate multiform emulsions for a wide range of applications with high throughput such as encapsulation, chemical synthesis, and biochemical assays. ${ }^{13,14}$ Generally, droplet-based microfluidic systems create discrete volumes that serve as confined spaces serving as microreactors, microcages, and so forth. The size of the generated droplets is mainly controlled by the fluid viscosity, flow rate, interfacial tension between the immiscible phases, flow geometry, and surface wettability of the microfluidic channels used for droplet generation. ${ }^{13,15,16}$ On downscaling to the microscale level, the high surface-to-volume ratio causes an increasing role of interfacial properties either between the immiscible fluidic phases coexisting in a microchannel or between the fluidic phase and the channel wall. ${ }^{13,17}$ Controlling the surface wettability of the microfluidic channel is therefore important when creating either $\mathrm{W} / \mathrm{O}$ or $\mathrm{O} / \mathrm{W}$ emulsion droplets or for the formation of double or multiple emulsions. W/O droplets are generally formed in a hydrophobic microchannel wetting the oil phase, whereas $\mathrm{O} / \mathrm{W}$ droplets can be obtained in a hydrophilic channel. ${ }^{13,18}$ To form a double emulsion, a two-

Received: February 19, 2019

Accepted: April 15, 2019

Published: April 15, 2019 
step process $^{19,20}$ or local modification of microfluidic channels ${ }^{21-23}$ is required to form two droplet generators with opposite wettability. ${ }^{24,25}$

Microfluidic devices are commonly fabricated in silicon, glass or polymeric materials such as polydimethylsiloxane (PDMS), cyclic olefin copolymer or poly(methyl methacrylate) using standard photolithography, soft-lithography, mechanical milling, or laser writing methods. ${ }^{26}$ Among these materials, PDMS-based microfluidic devices are especially popular, primarily due to their ease of fabrication, optical transparency, biological inertness, non-toxicity and gas permeability. ${ }^{27,28}$ However, because of its intrinsic hydrophobicity, PDMS encounters significant limitations in producing stable $\mathrm{O} / \mathrm{W}$ emulsion droplets. The microchannel surface must be treated to be hydrophilic to ensure effective wetting to the continuous aqueous solution, and the result of this treatment should be stable in time. ${ }^{29}$

Various surface modification methods have been investigated to achieve surface wettability control of PDMS microfluidic devices. Plasma oxidation is by far the most commonly used method which oxidizes the PDMS surface to create a hydrophilic silica surface layer; however, this method only leads to surface roughness and a temporary hydrophilic surface, as PDMS regains its original surface properties over time. ${ }^{30} \mathrm{UV}$ treatment facilitates much deeper modification of the PDMS surface without inducing cracks or mechanically weakening PDMS; however, this effect is also transient. ${ }^{31}$ Solgel treatments result in a glass-like layer on the PDMS surface; however, they are difficult to handle because of the complicated modification process which also limits their applications for simple geometries. ${ }^{32,33}$ Layer-by-layer (LbL) assembly deposits positively and negatively charged polymers onto the PDMS surface to successfully render it hydrophilic; however, the coating process is time-consuming and difficult to control because of its chemical sensitivity. ${ }^{28,29,34}$ Photoinitiated grafting has been applied for surface modification with advantages of allowing local modification of selected areas in channels with few steps. ${ }^{28,35}$ Various materials, such as acrylic acid, ${ }^{35-37}$ acrylamide, ${ }^{35}$ poly(ethylene glycol) $)^{38,39}$ and $\mathrm{N}$ isopropylacrylamide, ${ }^{40-42}$ have furthermore been reported to be grafted on the PDMS surface via photoinduced grafting, to render it hydrophilic.

Poly( $N$-isopropylacrylamide) (PNIPAAm) is well known as a thermoresponsive polymer and has been applied as thermoresponsive surface for cell culturing and harvesting. ${ }^{40,43}$ PNIPAAm changes its structure in response to temperature variation in aqueous solutions. The polymer chains of PNIPAAm form expanded structures in water at a temperature below the polymer lower critical solution temperature (LCST) of $32{ }^{\circ} \mathrm{C}$. At a temperature above the LCST, these chains form compact structures that can aggregate and precipitate from solution. ${ }^{44}$ As a result, the wettability of a PNIPAAm surface changes with the molecular structure in response to temperature variation, showing hydrophilic and hydrophobic properties at the temperature below and above LCST, respectively. ${ }^{45,46}$ The switchable wettability of PNIPAAm surfaces has been employed in various functional material ${ }^{47,48}$ and tissue engineering fields. . $^{4,49-51}$

In this work, PDMS-based microfluidic channels were modified by in-channel UV-induced grafting of NIPAAm to show thermally responsive wettability. The preparation of PDMS microfluidic devices and the surface modification process are demonstrated step-by-step. The properties and quality of the coatings at different stages was investigated by using total attenuated reflection infrared (ATR-IR) spectroscopy for chemical group characterization, contact angle (CA) measurement for surface wettability determination, and a scanning electron microscope (SEM) and atomic force microscope (AFM) for surface topology characterization. The effect of the UV initiating time on surface wettability modification was studied. The thermal wettability response of the prepared PNIPAAm-grafted PDMS (PNIPAAm-g-PDMS) was subsequently investigated, showing a large change of water CA by slightly varying temperature. Modification of a flat PDMS surface, entire microfluidic channels, and local parts of microfluidic channels have all been demonstrated. Emulsion droplets of $\mathrm{O} / \mathrm{W}, \mathrm{W} / \mathrm{O}, \mathrm{O} / \mathrm{W} / \mathrm{O}$, and $\mathrm{W} / \mathrm{O} / \mathrm{W}$ could be produced in a single microfluidic device via adjusting the temperature of the grafted surfaces. Such a flexible control over droplet types is much beneficial for applications such as the production of solid microspheres and core-shell microcapsules of various materials.

\section{EXPERIMENTAL}

2.1. Materials. $N$-Isopropylacrylamide (NIPAAm, CAS: 2210-255), benzophenone (CAS: 119-61-9), $\mathrm{NaIO}_{4}$ (CAS: 7790-28-5), benzyl alcohol (CAS: 100-51-6), and ethoxylated trimethylolpropane triacrylate (ETPTA) were all obtained from Sigma-Aldrich (Shanghai, China). 2-Dimethoxy-2-phenylacetophenone (Heowns Co., Tianjin, China) was used as a photoinitiator. Deionized (DI) water (18.2 $\mathrm{M} \Omega$ at $25^{\circ} \mathrm{C}$ ) was prepared using a Milli-Q Plus water purification system (Milli-Q Plus water purification, Sichuan Wortel Water Treatment Equipment Co. Ltd, Sichuan, China). Negative photoresist SU-8 3050 and developer solution were purchased from MicroChem (MA, USA) for fabricating the silica mold with designed microchannels. Positive photoresist SUN-120P (Suntific Microelectronic Materials Co. Ltd, Weifang, China) was used for fabricating the indium tin oxide (ITO) patterns. The PDMS (Sylgard 184) package was purchased from Dow Corning Corporation (Midland-Michigan, USA) and was used for fabricating the microfluidic chip. ITO-coated glass slides (Shenzhen Laibao Hi-tech Co. Ltd., China) were used to fabricate ITO heaters. Silver conductive epoxy was purchased from MG Chemicals (Surrey, BC, Canada).

2.2. PDMS Device Fabrication. Designed microchannel patterns were transferred on a SU-8 (negative photoresist) layer spin-coated on a silicon wafer (Lijing Optoelctronics Co. Ltd, Suzhou, China), to serve as master mold using the standard photolithography technique. ${ }^{52}$ PDMS prepolymer and curing agent were mixed using a stirring machine at a mass ratio of $10: 1$ and then degassed in a vacuum chamber. The mixture was then casted onto the master mold and thermally cured in a heater at $90{ }^{\circ} \mathrm{C}$ for $0.5 \mathrm{~h}$. The PDMS replica with designed channel patterns was then peeled from the silicon master and cut into the predesigned size. The PDMS with designed channel patterns was bonded with a block of $1 \mathrm{~mm}$ thick flat PDMS layer after $\mathrm{O}_{2}$ plasma bonding in a plasma cleaner (PDC-002, Mycro Technologies Co., Ltd, Beijing, China) at $27 \mathrm{~W}$ power for $3 \mathrm{~min}$ to form a microfluidic chip. The schematic drawing of this process is shown in Figure S1.

2.3. ITO Heater Fabrication. A patterned ITO heater was fabricated by standard photolithography and a wet-etching process, as shown in Figure S2. ITO-glass was first ultrasonically washed with DI water and $99.5 \%$ ethanol, cleaned with $\sim 4.0 \mathrm{wt} \%$ alkaline cleaning agent, and then rinsed with DI water and blow dried using a nitrogen gun. Afterward, the cleaned ITO-glass was spin-coated by a photoresist (SUN-120P) layer and then exposed to UV (27 mW/ $\mathrm{cm}^{2}$ ) for $30 \mathrm{~s}$ through a patterned transparent photomask. The heater patterns were developed in a $4.0 \mathrm{wt} \% \mathrm{KOH}$ solution. ITO etching was performed by immersing the photoresist patterned slides in an aqueous solution containing $\mathrm{HNO}_{3}, \mathrm{HCl}$, and $\mathrm{H}_{2} \mathrm{O}$ (with volume ratio of $3: 50: 50)$ at $50{ }^{\circ} \mathrm{C}$ for $1.5 \mathrm{~min}$. SUN-120P was then removed 


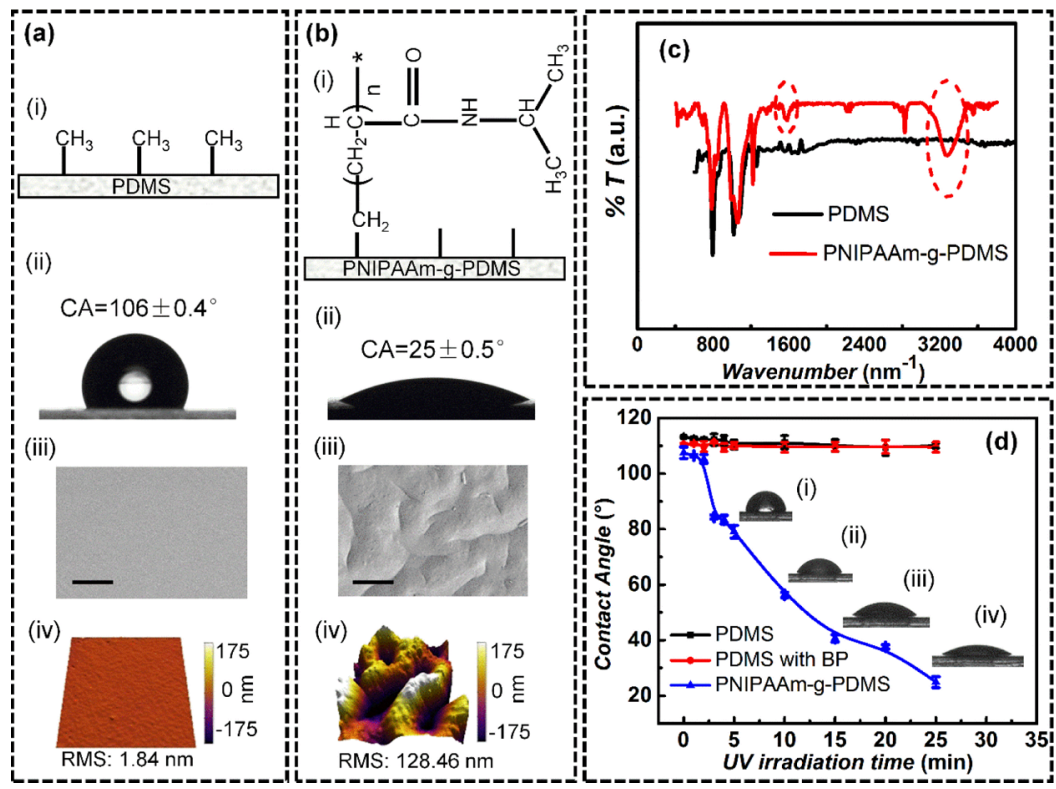

Figure 1. Mechanism and characterization of grafting PNIPAAm on the PDMS surface. (a) Bare PDMS surface: (i) chemical groups on PDMS surface, (ii) water CA on bare PDMS surface, (iii) SEM image of a bare PDMS surface, and (iv) AFM image of a bare PDMS surface (rms = 1.84 nm). (b) PNIPAAm-g-PDMS surface: (i) chemical groups on PNIPAAm-grafted PDMS surface, (ii) water CA on a PNIPAAm-g-PDMS surface, (iii) SEM image of a PNIPAAm-g-PDMS, and (iv) AFM image of a PNIPAAm-g-PDMS surface (rms = $128.46 \mathrm{~nm}$ ). This PNIPAAm-g-PDMS was prepared at 25 min UV irradiation. (c) FT-IR spectra of the PDMS and PNIPAAm-g-PDMS. (d) Effect of the UV irradiation time on water CAs on bare PDMS, PDMS treated with benzophenone-acetone solution (PDMS with BP), and PNIPAAm-g-PDMS surfaces at $25{ }^{\circ} \mathrm{C}$, with (i-iv) being the PNIPAAm-g-PDMS surfaces prepared at UV irradiation time of 5, 10,15, and 25 min, respectively. All scale bars denote $10 \mu$ m.

by dissolving in ethanol. The electrical conductors were fabricated by coating the patterned heater with $5 \mathrm{~nm}$ of chromium followed by sputtering $35 \mathrm{~nm}$ of gold (AJA International. ATC Orion 8, USA) covered with a shadow mask.

\section{RESULTS AND DISCUSSION}

3.1. UV-Induced Surface Grafting of NIPAAm on PDMS Mediated by Benzophenone. UV-induced grafting of PNIPAAm was conducted according to the method reported by Ebara et al. ${ }^{40}$ Benzophenone, a photosensitizer, was dissolved in acetone (20.0 wt \%). One millimeter thick flat PDMS film was immersed in benzophenone-acetone solution for $10 \mathrm{~min}$ at room temperature to let the photosensitizer be fully absorbed into PDMS. Afterward, the piece of the PDMS film was thoroughly rinsed with ethanol and water. The other layer of the PDMS film with a thickness of $3 \mathrm{~mm}$ was then prepared. Two PDMS films were aligned and integrated together with a gap distance of $60 \mu \mathrm{m}$ controlled by a PDMS spacer with a known thickness. The monomer solution containing NIPAAm (10.0 wt \%), $\mathrm{NaIO}_{4}(0.5 \mathrm{mM})$, and benzyl alcohol $(0.5 \mathrm{wt} \%)$ was then filled into the gap between the two PDMS films by capillary filling. This device was subsequently placed in an oven with a UV irradiator $(210 \mathrm{~W}$, $365 \mathrm{~nm}$, Intelliray 600, Uvitron International Inc., USA) to graft the PNIPAAm onto the PDMS surfaces and then was immersed and washed in DI water to remove residual monomer and polymer. An ice-water bath was then used to keep the temperature of the monomer solution below the LCST of NIPAAm.

Bare PDMS consists of repeating $-\mathrm{OSi}\left(\mathrm{CH}_{3}\right)_{2}-$ units on surface. The $-\mathrm{CH}_{3}$ groups result in poor surface wettability to water with a CA of about $106 \pm 0.4^{\circ}$. UV irradiation induces the generation of free radicals which cause NIPAAm monomers to polymerize on the treated PDMS surface. ${ }^{35,37}$ Figure 1a,b illustrates the initial PDMS surface and PDMS with the grafted PNIPAAm layer after UV irradiation. ${ }^{53}$ In Figure 1c, the Fourier transform infrared (FT-IR) spectrum (Vertex70 ATR-FTIR spectrometer, Bruker, Germany) of the PNIPAAm-g-PDMS surface shows a new absorbance peak at around $1660 \mathrm{~nm}^{-1}$, compared with the spectrum of the bare PDMS surface. This new peak can be assigned to the $\mathrm{C}=\mathrm{O}$ stretching vibration of the amide group. Another high and broad peak at $\sim 3300 \mathrm{~nm}^{-1}$ can be attributed to the $\mathrm{N}-\mathrm{H}$ stretching vibration. Both features indicate that PNIPAAm has been grafted on the PDMS surface.

Surface wettability was characterized by using a CA interfacial tension meter (OCA Pro15, Dataphysics, Germany). Flat PDMS surfaces with and without grafted polymer were prepared using the same protocol. Images of CA measurements were captured at $10 \mathrm{~s}$ after a water drop was placed onto the surface. A CA of $\sim 106^{\circ}$ demonstrated the hydrophobicity of the bare PDMS surface, as shown in Figure 1aii. However, when PNIPAAm was grafted onto PDMS surface, a CA of $\sim 25^{\circ}$ was obtained (Figure 1bii), suggesting a hydrophilic surface. SEM images (Figure 1aiii,biii) showed an increase in the surface roughness of PNIPAAm-g-PDMS compared with bare PDMS; and the root-mean-square (rms) roughness from AFM images (Figure laiv,biv) was 128.46 and $1.84 \mathrm{~nm}$ for PNIPAAm-g-PDMS and bare PDMS surfaces, respectively.

The UV irradiation time was investigated to determine its influence on the grafted surface wettability. As shown in Figure 1d, CAs on the PNIPAAm-g-PDMS surfaces changed with the UV irradiation time. At the UV irradiation time of $<3 \mathrm{~min}$, CA did not change significantly. For UV irradiation time of $>3$ min, CA decreased with irradiation time, with CAs of $84 \pm 0.7$, $79 \pm 2,56 \pm 1,40.7 \pm 1,37.5 \pm 0.7$, and $25 \pm 2.7^{\circ}$ obtained at the irradiation time of $3,5,10,15,20$, and $25 \mathrm{~min}$, respectively. CAs on the surfaces of bare PDMS and PDMS pretreated with benzophenone-acetone solution (PDMS with BP) did not 


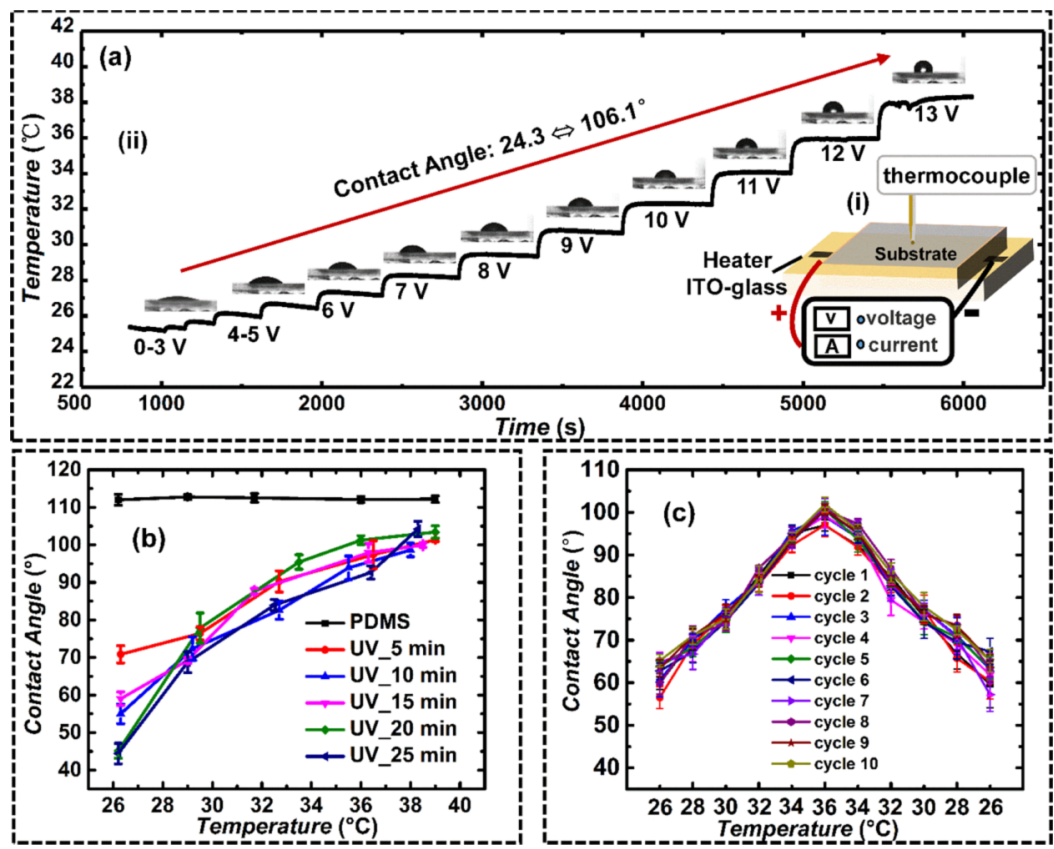

Figure 2. Thermoresponsive surface wettability of PNIPAAm-g-PDMS. (a) Water CAs on a PNIPAAm-g-PDMS surface (prepared by 25 min UV irradiation) changing with temperature: (i) schematic drawing of the temperature control by an ITO heater and measurement by a thermocouple device, and (ii) PDMS surface temperature versus heating time at different applied voltages, and the water CAs on a PNIPAAm-g-PDMS surface at different temperature. (b) Effect of temperature on PNIPAAm-g-PDMS surface wettability. Samples were prepared using the same process but varying UV irradiation time of 5, 10, 15, 20, and $25 \mathrm{~min}$. (c) Reversibility and repeatability of thermoresponsive surface wettability of the PNIPAAm-g-PDMS film. The error bars represent the standard deviation calculated from five repeated experiments for each data point.

show obvious changes with the UV irradiation time in the range of $0-25 \mathrm{~min}$.

In general, a major difficulty for surface wettability coating is its stability when exposed to air and water, especially for polymer coatings, either due to the slow process of the movement of hydrophobic groups to the polymer surface or due to the low molecular weight polymer from the bulk to the surface. ${ }^{35}$ Therefore, in this work, we have also investigated the long-term stability of PNIPAAm-g-PDMS after UV irradiation times of 5, 10, 15, 20, and 25 min by measuring the CAs after days and weeks of storage under environmental conditions in air, with the results shown in Figure S3. Typically, the CA slightly (with about $10^{\circ}$ ) increased with the storage time in several days after being prepared and became stable after 1 week. When the UV irradiation time was longer than $10 \mathrm{~min}$, the surface became stable after 4 weeks storage with CAs typically lower than $70^{\circ}$.

\subsection{Thermally Responsive Surface Wettability of} PNIPAAm-g-PDMS. A glass substrate coated with ITO was used as an electrical heater with a resistance of $330 \Omega$. An ITO heater was clamped with a PDMS substrate to control the PDMS surface temperature via applying a voltage for a certain duration of time. The exact temperature on a PNIPAAm-gPDMS film was measured by using a Type-T Thermocouple (Copper/Constantan), with the data collected by a data acquisition system (Keithley Series 2700, USA), as shown in Figure 2ai. Patterning of the ITO heater was realized by standard photolithography. A copper wire was glued to the heat conductor using silver conductive epoxy. Figure 2aii shows the sequential CA change on a PNIPAAm-g-PDMS sample surface (prepared with $25 \mathrm{~min}$ UV irradiation) with temperature controlled by the ITO heater.

On a PNIPAAm-g-PDMS sample surface prepared by 25 min UV initiation, the water CA varied from 24.3 to $106.1^{\circ}$ when the surface temperature increased from 25 to $38{ }^{\circ} \mathrm{C}$ (Figure 2a). Figure $2 \mathrm{~b}$ shows the water $\mathrm{CA}$ varying with temperature on PNIPAAm-g-PDMS surfaces prepared with different UV irradiation times of 5, 10, 15, 20, and $25 \mathrm{~min}$. In contrast to the bare PDMS, all samples of PNIPAAm-g-PDMS showed thermally switchable surface wettability with the corresponding CAs in the ranges of 70.8-101.3, 55-98.6, 59-100, 45.1-103.4, and 44.4-104 ${ }^{\circ}$. At the temperature of 32 ${ }^{\circ} \mathrm{C}$ (LCST of NIPAAm), the CA on PNIPAAm-g-PDMS surface was about $80^{\circ}$, and the surface became hydrophobic at a temperature above $34{ }^{\circ} \mathrm{C}$. These observations show that a surface with a thermally responsive wettability has been obtained. This surface wettability can be reversibly varied between hydrophilic and hydrophobic over a wide range of CAs.

Repeatable and reversible temperature control over the surface wettability was also investigated by heating and cooling the same sample for multiple cycles. PNIPAAm-g-PDMS films were prepared under UV irradiation times of 10, 15, 20, and 25 min. We chose a temperature range from 25 (room temperature) to $36{ }^{\circ} \mathrm{C}$ (the PNIPAAm-g-PDMS becomes hydrophobic according to Figure $2 b$ ). Figure $2 c$ shows the surface wettability changes of a PNIPAAm-g-PDMS film (10 min UV irradiation) over 10 cycles of heating and cooling. At temperature below $34{ }^{\circ} \mathrm{C}$, the PNIPAAm-g-PDMS surfaces were hydrophilic with water CAs of $\angle 90^{\circ}$, while they became hydrophobic with CAs of $>90^{\circ}$ when heated above $34{ }^{\circ} \mathrm{C}$. Experimental results show excellent reversibility in wettability control over the 10 heating-cooling cycles. The slight increase in initial CAs from $59.9 \pm 1.4$ to $65.1 \pm 1.5^{\circ}$ after several heating-cooling cycles, may be attributed to contaminants ${ }^{54}$ or the precipitation of benzophenone during the solvent evaporation in the heating process. Figure S4 shows results for PNIPAAm-g-PDMS films prepared under the UV irradiation 


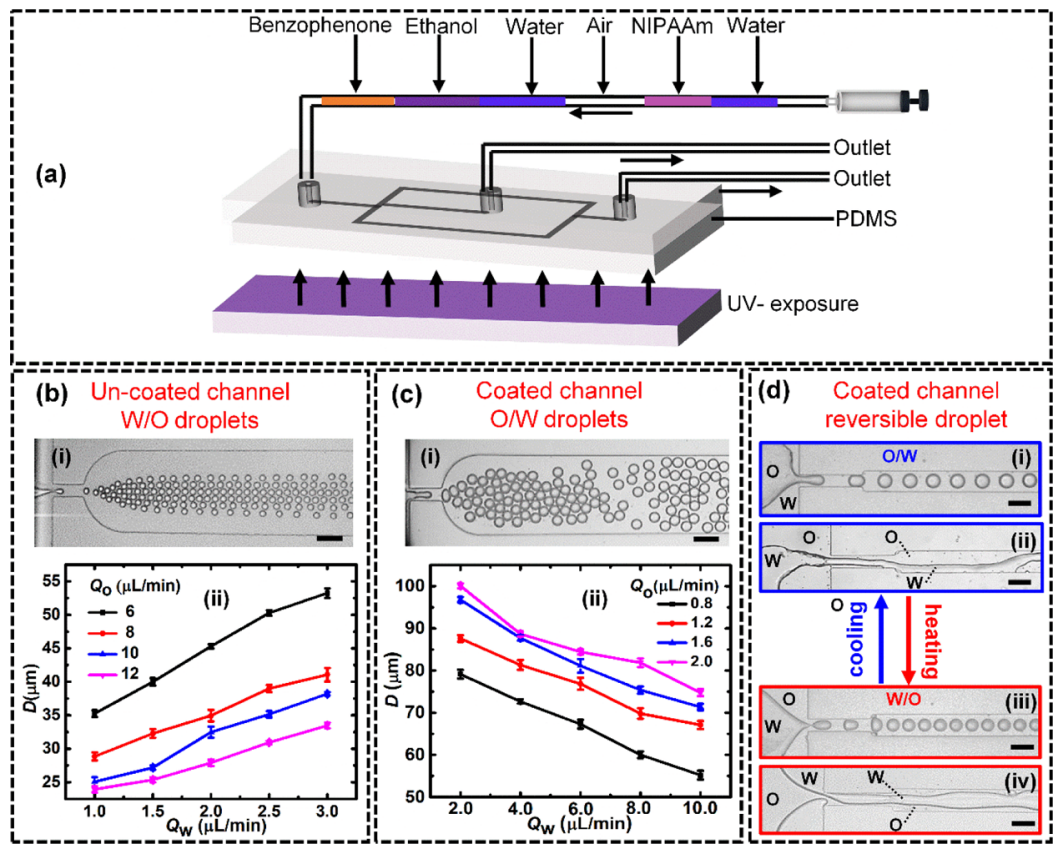

Figure 3. Uncoated and coated PDMS microfluidic chips for tunable droplet types and stratified flows via wettability control. (a) Schematic drawing of the in-channel surface treatment process. (b) W/O droplets generated in uncoated bare PDMS channels with (i) a snapshot of the droplet generation at the flow-focusing junction and (ii) the dependence of droplet diameter $(D)$ on water-phase flow rate $\left(Q_{w}\right)$ at constant oil flow rate $\left(Q_{0}\right)$. (c) O/W droplets generated in coated PDMS channels with (i) a snapshot of the droplet generation at the flow-focusing junction and (ii) the curves of $D$ vs $Q_{w}$ at constant $Q_{0}$. The error bars represent the standard deviation calculated from 200 droplets for each data point. (d) Reversible (i) O/W $\left(Q_{w}=2.0 \mu \mathrm{L} / \mathrm{min}, Q_{0}=0.5 \mu \mathrm{L} / \mathrm{min}\right)$ and (iii) W/O $\left(Q_{0}=5.0 \mu \mathrm{L} / \mathrm{min}, Q_{w}=2.0 \mu \mathrm{L} /\right.$ min $)$ droplet formation controlled by cooling $\left(25{ }^{\circ} \mathrm{C}\right)$ and heating $\left(36{ }^{\circ} \mathrm{C}\right)$ the PNIPAAm-g-PDMS microfluidic channels, obtained by the thermoresponsive surface, with the corresponding (ii) $\mathrm{O} / \mathrm{W}$ and (iv) W/O in-parallel flows, by using surfactants (SDS and Span 80) with opposite wettability to the channel surface. All channel depths are $60 \mu \mathrm{m}$. The scale bars denote $100 \mu \mathrm{m}$.

time of 15,20 , and $25 \mathrm{~min}$, showing a similar trend as that in Figure $2 c$. The hydrophilicity of prepared PNIPAAm-g-PDMS surfaces is thus conserved for multiple heating-cooling cycles, and the transition temperature from hydrophilic to hydrophobic remains at approximately $34{ }^{\circ} \mathrm{C}$.

3.3. In-Channel Surface Treatment in Microfluidic Devices for Reversible Droplet and Two-Phase Flow Types. At the micro- and nano-scale, the emulsion droplet type depends critically on the preferential wetting of the channel walls by the continuous phase, and the change of microchannel surface wettability can cause emulsion inversion. ${ }^{55}$ By using the same grafting process as for the open surface, an in-channel surface coating was realized by sequentially flowing the corresponding compounds through the selected channel areas. Figure $3 a$ shows the schematic of the in-channel surface treatment process. Benzophenone solution (20.0 wt \% in acetone) was pumped into the bare PDMS channel for $3 \mathrm{~min}$ to cause benzophenone to be fully absorbed in PDMS. The channel was then washed extensively with ethanol and water and flushed with air to dry it. Afterward, NIPAAm monomer solution containing NIPAAm (10.0 wt \% in water), $\mathrm{NaIO}_{4}(0.5 \mathrm{mM})$, and benzyl alcohol (0.5 wt \%) was loaded into the channel. Intensive UV light (210 W, $365 \mathrm{~nm}$, Intelliray 600, Uvitron International Inc., USA) was then applied to initiate the cross-linking of the methyl groups on the PDMS and NIPAAm to obtain PNIPAAm-g-PDMS microfluidic channels. The channels were then flushed with DI water and kept filled with DI water by connecting the tubing to a water reservoir to avoid drying. The preferential wetting toward water or oil at different temperature has been investigated by measuring the water-in- oil CAs on a PNIPAAm-g-PDMS surface, as summarized in Table S1. The grafted surface shows preferential to water and oil phases at the temperature below and beyond $32{ }^{\circ} \mathrm{C}$ (LCST of PNIPAAm), respectively. This shows obviously the possibility for controlling emulsion droplet types generated in channels by varying temperature.

In general, $\mathrm{W} / \mathrm{O}$ emulsion droplets are produced in bare PDMS channels because of its hydrophobicity. As shown in Figure $3 \mathrm{~b}$, water droplets in mineral oil solution (containing 3.0 wt \% Span 80) were generated in a flow-focusing PDMS chip (Figure 3bi), where the droplet size was controlled by adjusting the disperse water-phase flow rate $\left(Q_{w}\right)$ and continuous oil-phase flow rate $\left(Q_{0}\right)$. In this experiment, the values of $Q_{w}$ were fixed at 1.0, 1.5, 2.0, 2.5, and $3.0 \mu \mathrm{L} / \mathrm{min}$ with $Q_{0}$ being increased from 6.0 to $12.0 \mu \mathrm{L} / \mathrm{min}$. Obtained $\mathrm{W} / \mathrm{O}$ emulsion droplets under different flow rate conditions are shown in Figure 3bii, suggesting that the generated droplet diameter $(D)$ increases with the increase of $Q_{w}$ and decreases with the increase of $Q_{0}$. Monodispersed water droplets are obtained with diameters ranging from 23 to $54 \mu \mathrm{m}$ and corresponding relative standard deviations (RSDs) less than $3.0 \%$.

When the hydrophobic PDMS surface was grafted with a layer of PNIPAAm, a hydrophilic surface was obtained (Figure 1b). As a result, the water phase preferentially wetted the channel surface instead of oil, and consequently, the formation of oil droplets in water phase was achieved, as demonstrated in Figure 3c. Mineral oil droplets were formed in aqueous solution containing $3.0 \mathrm{wt} \%$ sodium dodecylsulfate (SDS) in PNIPAAm-grafted PDMS chips (Figure 3ci). The generated droplet size could be tuned by varying the continuous water 
$\left(Q_{w}\right)$ and dispersed oil $\left(Q_{0}\right)$ flow rates in the same device, increasing with the increase of $Q_{0}$ and decreasing with the increase of $Q_{w}$, as shown in Figure 3cii. In this experiment, $Q_{0}$ was fixed at $0.8,1.2,1.6$, and $2.0 \mu \mathrm{L} / \mathrm{min}$ with $Q_{\mathrm{w}}$ increased from 2.0 to $10.0 \mu \mathrm{L} / \mathrm{min}$. The obtained oil droplet diameter was in the range of 55-101 $\mu \mathrm{m}$ with RSDs of $<2 \%$. To evaluate the coating stability, the same device was reused several times over 1 month. Similar O/W droplet generation process and flow regimes were obtained without obvious changes.

The thermoresponsive wettability in the microfluidic channels was then evaluated by heating-cooling cycles. An ITO heater was placed underneath the microfluidic device to control the temperature via the applied voltage. As shown in Figure $2 b$, the hydrophilic PNIPAAm-g-PDMS surface could be efficiently changed to hydrophobic by heating above $34^{\circ} \mathrm{C}$. In the experiment, the surface was heated up to $\sim 36{ }^{\circ} \mathrm{C}$ to ensure a hydrophobic channel surface. At the room temperature of $\sim 25{ }^{\circ} \mathrm{C}$, the PNIPAAm-grafted PDMS channels behaved hydrophilic, making the water phase acting as continuous phase. As shown in Figure 3di, silicon oil droplets were produced in an aqueous solution containing $3.0 \mathrm{wt} \%$ SDS as the surfactant. Also, when using silicon oil containing 2.0 wt \% Span 80 as continuous phase (a more hydrophobic surfactant), water droplets were not formed which can be ascribed to the fact that the oil phase is disfavored by the channel walls (Figure 3dii). Instead, as we expect, because of the competition between the interfacial preference of forming W/O curvature caused by the Span 80 surfactant and the solid surface wettability to the water phase, an oil-in-water in-parallel flow was still obtained but without droplet formation. When the channel is heated up to $36^{\circ} \mathrm{C}$, the coated channel surface becomes hydrophobic and the oil phase wets the channel surface. As a result, water droplets in silicon oil containing 2.0 wt \% Span 80 were formed (Figure 3diii). Because of the same reason as described above, in the hydrophobic channel at high temperature, a water-in-oil in-parallel flow was observed as shown in Figure 3div. In summary, the continuous and dispersed phases could be changed at will by thermally switching the surface wettability in a reversible way.

3.4. Surface Wettability Determined Stable and Instable Droplet Formation with the Same Fluidic Compositions in the Same Microfluidic Device. As shown above, stable $\mathrm{O} / \mathrm{W}$ and $\mathrm{W} / \mathrm{O}$ droplets could be stably generated in thermally switched hydrophilic and hydrophobic channels, respectively, by applying suitable hydrophilic and hydrophobic surfactants in the water and oil phases. However, when a two-phase fluidic system is used of a constant composition that can generate both $\mathrm{O} / \mathrm{W}$ and $\mathrm{W} / \mathrm{O}$ droplets, the microfluidic channel surface wettability becomes the key factor controlling the droplet creation processes and thus the emulsion types. Thus, a microfluidic channel with tunable surface wettability can help to find out the boundaries of the regimes where $\mathrm{O} / \mathrm{W}$ and $\mathrm{W} / \mathrm{O}$ droplets are created, as well as the regimes where stable and instable droplet formation processes occur. To investigate this possibility, we chose a twophase fluidic system with a hydrophilic surfactant in water and a hydrophobic surfactant in oil. An aqueous solution containing $4.0 \mathrm{wt} \%$ PVA (surfactant) and $30.0 \mathrm{wt} \%$ glycerol was used as the aqueous phase, and HFE-7500 containing 2.0 wt \% PFPE-PEG600 (surfactant) was applied as the oil phase. From this fluidic system, both $\mathrm{O} / \mathrm{W}$ and W/O droplets could be generated by using conventional mechanical agitation. In this work, we could dynamically adjust the surface wettability by heating and cooling the microfluidic devices with the PNIPAAm-g-PDMS channel surface. As a result, stable or instable $\mathrm{O} / \mathrm{W}$ and stable or instable $\mathrm{W} / \mathrm{O}$ droplets could be obtained at choice in a single microfluidic device by regulating the temperature, as shown in Figure 4. At room temperature,

(a)

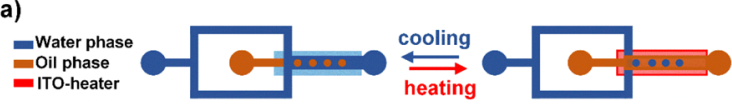

(b)

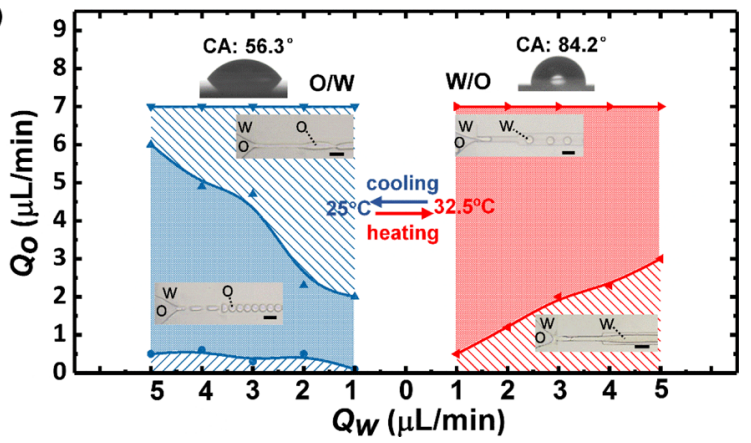

Figure 4. Reversible tuning $\mathrm{O} / \mathrm{W}$ and $\mathrm{W} / \mathrm{O}$ at droplet and stratified flows by thermoresponsive surface wettability in one microfluidic device using the same fluidic system. (a) Diagrammatic drawing of dynamically reversible emulsion droplet generation. (b) Stable and instable droplet formation in a heating and cooling process. Blue and red areas are $\mathrm{O} / \mathrm{W}$ and $\mathrm{W} / \mathrm{O}$ flow types, being reversible in a cooling and heating cycle. CAs of $56.3^{\circ}$ and $84.2^{\circ}$ represent hydrophilic and hydrophobic surface wettability at the temperature of 25.0 and 32.5 ${ }^{\circ} \mathrm{C}$, respectively. The microfluidic channels were coated with PNIPAAm under UV irradiation time of $10 \mathrm{~min}$. The water and oil phases were aqueous solution containing $4.0 \mathrm{wt} \%$ PVA (surfactant) and 30.0 wt \% glycerol, and HFE-7500 containing $2.0 \mathrm{wt} \%$ PFPEPEG600 (surfactant). All scale bars denote $50 \mu \mathrm{m}$.

the water phase served as continuous phase because the PNIPAAm-coated channel wall is hydrophilic (with water CA of $56.3^{\circ}$ ), and $\mathrm{O} / \mathrm{W}$ emulsion droplets were obtained at a large range of oil and water flow rates. When the oil (disperse phase) flow rate was too high to form droplets, stratified flow was obtained with the water phase still wetting the channel walls. However, when heating up to $32.5^{\circ} \mathrm{C}$, the water CA changes to $84.2^{\circ}$ and the oil phase started to wet the channel wall and hence became the continuous phase. As a result, W/O emulsion droplets were obtained. Moreover, in this case, we have observed a stratified flow when the water flow rate increased above a threshold value, with the oil phase still wetting the channel walls. In this way, it proves that the surface wettability determines emulsion types generated inside the microfluidic device no matter how inner and outer phases are introduced. Such a surface wettability-induced dynamic reversible emulsion process will be highly useful for studying the phase inversion mechanism, which has applications in the food and cosmetics industry where phase inversion is necessary or instead needs to be avoided. ${ }^{11,12}$

\subsection{Controllable Double Emulsion Droplet Prepara-} tion in Microfluidic Devices by Local Temperature Control. Double emulsions of either $\mathrm{W} / \mathrm{O} / \mathrm{W}$ and $\mathrm{O} / \mathrm{W} / \mathrm{O}$ types can encapsulate various contents as cores (inner phase) via forming shells (middle phase) to form core-shell structures and have found a variety of applications including microcapsule fabrication, ${ }^{56}$ vesicle preparation, ${ }^{57}$ drug delivery, ${ }^{58,59}$ chemical synthesis, ${ }^{60}$ and single cell screening. ${ }^{61}$ Controlled 


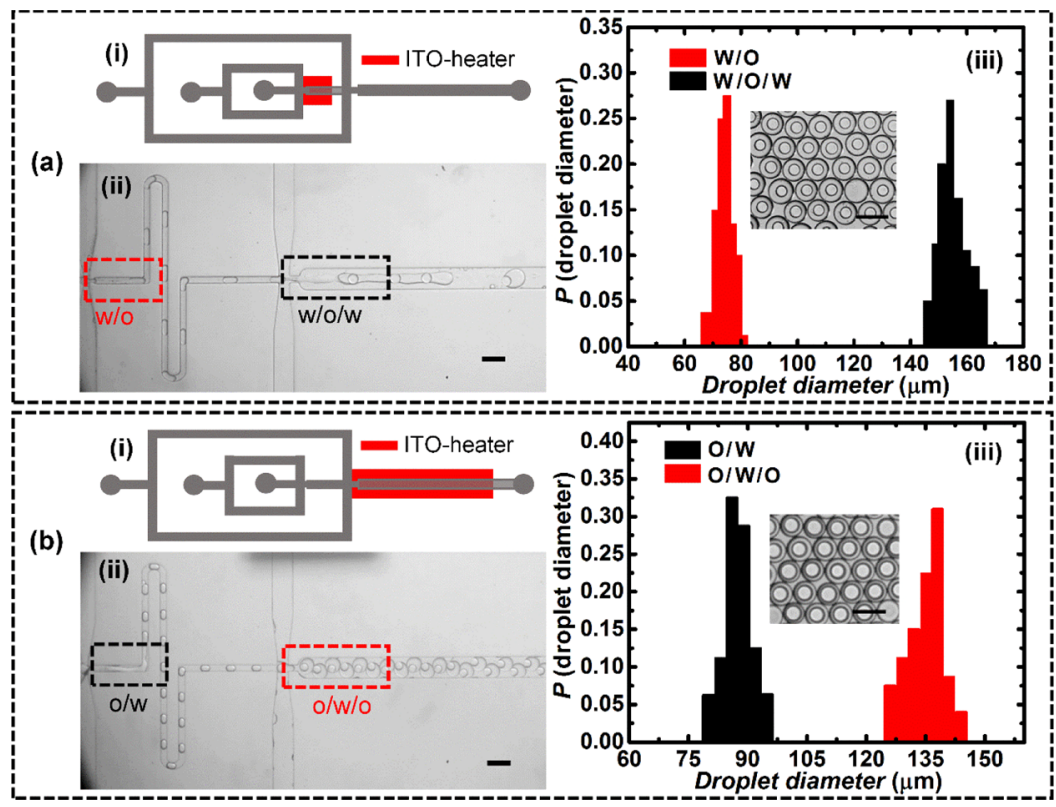

Figure 5. Local surface wettability control via temperature and corresponding double emulsion droplets formation in the same microfluidic device with PNIPAAm-g-PDMS surface. (a) Local heating at the first FFD section for producing W/O/W double emulsion droplets: (i) schematic drawing of selectively heating the first FFD section, (ii) formation of $\mathrm{W} / \mathrm{O}$ and $\mathrm{W} / \mathrm{O} / \mathrm{W}$ droplets at the first and second FFD junctions, and (iii) collected droplets and droplet size distribution. (b) Local heating at the second FFD section for producing O/W/O double emulsion droplets: (i) schematic drawing of selectively heating the second FFD section, (ii) formation of $\mathrm{O} / \mathrm{W}$ and $\mathrm{O} / \mathrm{W} / \mathrm{O}$ droplets at the first and second FFD junctions, and (iii) collected droplets and droplet size distribution. All scale bars denote $200 \mu \mathrm{m}$. The droplet diameter distribution was calculated with a total droplet number of 200 .

fabrication of multiple emulsion droplets using microfluidics has been well developed in recent years. Generation of double emulsion droplets has been reported by using microfluidic devices of assembled glass capillaries ${ }^{60,61}$ or selectively modified microfluidic channels. ${ }^{23,62-64}$ Fixed emulsion types of either $\mathrm{W} / \mathrm{O} / \mathrm{W}$ or $\mathrm{O} / \mathrm{W} / \mathrm{O}$ could be produced in those devices once the microfluidic channels were prepared. Hereby, we demonstrate that the necessary surface wettability changes can also be obtained in a reversible and switchable manner in a single device by PNIPAAm-grafted surface and temperature control. For this purpose, microfluidic devices with two sections of flow-focusing junctions were selectively modified. Local modification of PDMS channels was enabled by selectively filling the channel section with air to block the BP solution and thus preventing PNIPAAm grafting to keep its hydrophobicity. As demonstrated in Figure S5ai,bi, PDMS microfluidic devices were selectively modified to obtain opposite wettability at the two consecutive flow-focusing device (FFD) sections. Figure S5 shows the corresponding formation of single and double emulsion droplets. The sequential untreated-treated and treated-untreated PDMS FFDs could produce $\mathrm{W} / \mathrm{O} / \mathrm{W}$ and $\mathrm{O} / \mathrm{W} / \mathrm{O}$ droplets, respectively. Here, the high contrast in surface wettability of the PDMS $\left(\mathrm{CA}=106^{\circ}\right)$ and PNIPAAm-g-PDMS $\left(\mathrm{CA}=25^{\circ}\right)$ is used for stably creating double emulsions.

The key advantage of the PNIPAAm material is its thermoresponsive property. Therefore, we investigated inchannel local surface wettability control by locally heating part of the microfluidic channels, as demonstrated in Figure 5. The two FFD sections were first treated to be hydrophilic by grafting a PNIPAAm layer. Under each FFD section, an ITO heater strip was placed to selectively control its surface temperature via an applied voltage, as shown in Figure 5ai,bi. In this way, the surface wettability of the two sections of FFD could be selectively manipulated with the same or opposite wettability with a water CA difference of about $80^{\circ}$.

To form $\mathrm{W} / \mathrm{O} / \mathrm{W}$ double emulsion droplets, the first (left) FFD section should be hydrophobic while the second (right) FFD section should be hydrophilic. The PNIPPAm-g-PDMS surface is hydrophilic at a temperature of $\sim 25{ }^{\circ} \mathrm{C}$. When the first FFD section was heated up to $36{ }^{\circ} \mathrm{C}$ while keeping the second FFD section at $\sim 25{ }^{\circ} \mathrm{C}$, the first and second FFD sections became hydrophobic and hydrophilic, respectively. Indeed, $\mathrm{W} / \mathrm{O} / \mathrm{W}$ double emulsion droplets were obtained, as presented in Figure 5aii. The inner, middle, and outer fluidic phases were DI water, FC-40 containing 2.0 wt \% PFPEPEG600 as surfactant, and aqueous solution containing $2.0 \mathrm{wt}$ $\%$ PVA and 15.0 wt \% glycerol, respectively, with the corresponding flow rates of $0.3,1.8$, and $14 \mu \mathrm{L} / \mathrm{min}$. On the other hand, $\mathrm{O} / \mathrm{W} / \mathrm{O}$ double emulsion droplets were successfully generated in the same device by just locally heating the second FFD section, as shown in Figure 5bii. The inner, middle, and outer fluidic phases were HFE-7500, aqueous solution containing 2.0 wt \% PVA and $15.0 \mathrm{wt} \%$ glycerol, and HFE-7500 containing 2.0 wt \% PFPE-PEG600 as the surfactant, respectively, with the corresponding flow rates of $0.5,1.5$, and $4.0 \mu \mathrm{L} / \mathrm{min}$. Collected droplets were analyzed using Image J software, as presented in Figure 5aiii,biii, both the single emulsion droplets produced at the first FFD junction and the double emulsion droplets generated at the second FFD junction show good monodispersity with RSD of $<7 \%$. Higher enwrapping efficiency (the portion of double emulsion droplets in all generated droplets) was obtained by adjusting the flow rates of the three phases.

3.6. Synthesis of Core-Shell Microcapsules via Double Emulsion Droplets as Templates. To demonstrate the usability and reliability of the thermoresponsive surface wettability of the PNIPAAm-g-PDMS, preparation of core- 
shell microcapsules in locally heated PNIPAAm-g-PDMS chips was investigated. To produce core-shell microcapsules, double emulsion droplets containing an inner liquid phase (core) and middle liquid phase (shell) in the outer liquid phase are prepared. In this work, we chose the aqueous solution containing $10.0 \mathrm{wt} \%$ glycol, the ETPTA with 2-dimethoxy2-phenylacetophenone, and the aqueous solution containing $4.0 \mathrm{wt} \%$ PVA and $30.0 \mathrm{wt} \%$ glycerol, as inner, middle, and outer phases, respectively. Monodispersed double emulsion droplets were obtained at the inner, middle, and outer flow rate ranges of $0.035-0.4,0.45$, and $8.0 \mu \mathrm{L} / \mathrm{min}$, respectively. Collected droplets were then exposed to UV light $(210 \mathrm{~W}, 365$ $\mathrm{nm}$, Intelliray 600, Uvitron International Inc., USA) for $20 \mathrm{~s}$ to polymerize the middle ETPTA phase to form a hard shell. Microcapsules with different shell thicknesses were obtained by varying the flow rate of the inner phase. Figure 6 shows the

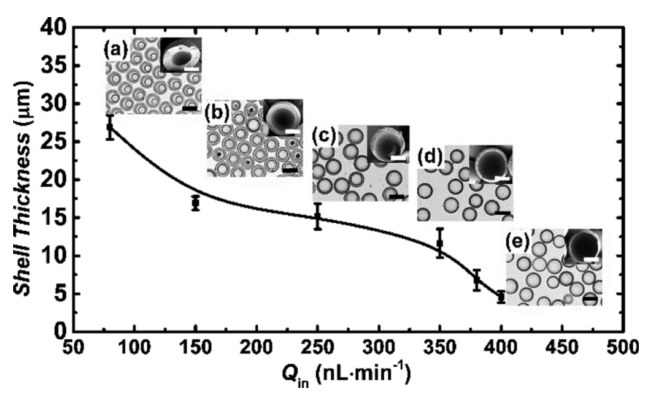

Figure 6. Controllable generation of double emulsion droplets for fabricating core-shell microcapsules with different shell thickness. (a-e) Microscopic images and SEM images of double emulsion droplets and core-shell microcapsules prepared at the inner flow rates of $0.08,0.15,0.28,0.35$, and $0.38 \mu \mathrm{L} / \mathrm{min}$, with the constant middle and outer phase flow rates of 0.45 and $8.0 \mu \mathrm{L} / \mathrm{min}$. The white scale bars denote $40 \mu \mathrm{m}$, and black scale bars denote $100 \mu \mathrm{m}$. The standard deviation was calculated from 100 core-shell capsules for each data point.

microscopic images of the double emulsion droplets and SEM images of the polymerized core-shell microcapsules with ETPTA shells at various thicknesses. The double emulsion droplets were obtained at the inner flow rates of $0.08,0.15$, $0.28,0.35$, and $0.38 \mu \mathrm{L} / \mathrm{min}$, while keeping the middle and outer flow rates at 0.45 and $8.0 \mu \mathrm{L} / \mathrm{min}$. Prior to SEM analysis, the microcapsules were ruptured between two glass slides to view the cross-sectional area. A $15 \mathrm{~nm}$ platinum layer was deposited onto the microcapsule surface before putting into the chamber of SEM. The microcapsule size and shell thickness in the range of $30-150$ and $1-40 \mu \mathrm{m}$ could be created by precisely tuning the fluidic properties and flow rates. Such microcapsules could be applied as microcontainers for encapsulating active agents for the preparation of fragrance retention powder, ${ }^{65}$ osmotic pressure triggered cavitation, ${ }^{66}$ photonic crystals, ${ }^{67}$ and so on. Moreover, because the wettability of the two FFD junctions could both be tuned between hydrophilic and hydrophobic to obtain high wettability contrast, various types of microcapsule materials could be synthesized using such a device, which would explore materials with more functionalities and thus expand their application fields.

\section{CONCLUSION}

In this work, a surface coating method based on photografting of the thermoresponsive polymer PNIPAAm onto PDMS has been investigated and applied in microfluidic devices. Bare hydrophobic PDMS surfaces became stably hydrophilic when grafted with PNIPAAm. A highly sensitive and reversible thermoresponsive wettability change of $7.4^{\circ} /{ }^{\circ} \mathrm{C}$ was achieved on the surfaces with the water CA changing between 25 and $106^{\circ}$, when varying the PNIPAAm-g-PDMS surface temperature between 25 and $38{ }^{\circ} \mathrm{C}$. The surface modification as well as thermal switching was found to be long-term stable under environmental conditions. A dynamically reversible generation of $\mathrm{O} / \mathrm{W}$ and $\mathrm{W} / \mathrm{O}$ emulsions was achieved via heating and cooling the same microfluidic device with PNIPAAm-grafted walls. Such a high wettability contrast with a switchable CA difference of $\sim 80^{\circ}$ brings high controllability over the single and double emulsion droplet formation using various fluidic compositions. Both $\mathrm{O} / \mathrm{W} / \mathrm{O}$ and $\mathrm{W} / \mathrm{O} / \mathrm{W}$ emulsion droplets can be generated either via selectively grafting part of PDMS channels or selectively heating the overall grafted microfluidic channels as designed, according to the opposite wettability of PDMS and PNIPAAm-g-PDMS surfaces and the thermoresponsive wettability of the PNIPAAm-g-PDMS surface. Dynamic tuning of the channel surface wettability enabled us, for the first time, to establish the regimes of $\mathrm{O} / \mathrm{W}$ droplet flow, $\mathrm{O} / \mathrm{W}$ stratified flow, $\mathrm{W} / \mathrm{O}$ droplet flow, and $\mathrm{W} / \mathrm{O}$ stratified flow, for the same two-phase fluidic system in the same microfluidic device. Core-shell microcapsules containing a fluidic inner core and a hard shell were also synthesized via the double emulsion droplet process, with a tunable core and shell thickness. Overall, we demonstrated a facile and rapid surface modification to prepare long-term stable hydrophilic PDMS surfaces with thermal wettability switching between hydrophilic and hydrophobic states. This method has the promise to broaden applications of PDMS-based microfluidic devices and can serve as a useful platform to study dynamical processes of reversible multiphase micro- and nano-fluidic phenomena on surfaces or in confined microspaces.

\section{ASSOCIATED CONTENT}

\section{Supporting Information}

The Supporting Information is available free of charge on the ACS Publications website at DOI: 10.1021/acsami.9b03160.

Schematic drawing of the fabrication process of the PDMS device; schematic drawing of the fabrication process of the patterned ITO heater; long-term stability of NIPAAm-coated PDMS channels; reversibility of thermoresponsive surface wettability on PNIPAAm-gPDMS films a under UV irradiation time of 15, 20, and 25 min; water-in-oil CA on PNIPAAm-g-PDMS surface at different temperature; and local modification of one of the FFD section via flow process and double emulsion droplets formation in corresponding devices (PDF)

\section{AUTHOR INFORMATION}

\section{Corresponding Author}

*E-mail: shuill@m.scnu.edu.cn. Phone: +86-20-3931-4813.

ORCID

Lingling Shui: 0000-0001-8517-1535

\section{Author Contributions}

L.L. and L.S. conceived the idea and scheme of this work. L.L. performed the experiments, analyzed the data, and prepared the manuscript draft, Z.Y. contributed to experimental setup buildup, partial data collection, and analysis. L.S. and J.C.T.E. contributed to data interpretation and manuscript preparation. 
All authors reviewed the manuscript. All contributed authors have given approval to the final version of this manuscript.

\section{Notes}

The authors declare no competing financial interest.

\section{ACKNOWLEDGMENTS}

We appreciate the financial support from the National Natural Science Foundation of China (61574065), Special Fund Project of Science and Technology Application in Guangdong (2017B020240002), Science and Technology Planning Project of Guangdong Province (2016B090906004), and the Cultivation Project of National Engineering Technology Center of Optofluidic Materials and Devices (2017B090903008). This work has also been partially supported by PCSIRT project no. IRT_17R40, the National 111 Project, and the MOE International Laboratory for Optical Information Technologies.

\section{REFERENCES}

(1) Rajaei-Sharifabadi, H.; Greene, E.; Piekarski, A.; Falcon, D.; Ellestad, L.; Donoghue, A.; Bottje, W.; Porter, T.; Liang, Y.; Dridi, S. Surface Wetting Strategy Prevents Acute Heat Exposure-Induced Alterations of Hypothalamic Stress- and Metabolic-Related Genes in Broiler Chickens. J. Anim. Sci. 2017, 95, 1132-1143.

(2) Charles-Orszag, A.; Tsai, F.-C.; Bonazzi, D.; Manriquez, V.; Sachse, M.; Mallet, A.; Salles, A.; Melican, K.; Staneva, R.; Bertin, A.; Millien, C. Adhesion to Nanofibers Drives Cell Membrane Remodeling through One-Dimensional Wetting. Nat. Commun. 2018, 9, 4450.

(3) Meskini-Vishkaee, F.; Mohammadi, M. H.; Neyshabouri, M. R. Revisiting the Wet and Dry Ends of Soil Integral Water Capacity Using Soil and Plant Properties. Soil Res. 2018, 56, 331-345.

(4) Du, X.; Wang, J.; Cui, H.; Zhao, Q.; Chen, H.; He, L.; Wang, Y. Breath-Taking Patterns: Discontinuous Hydrophilic Regions for Photonic Crystal Beads Assembly and Patterns Revisualization. ACS Appl. Mater. Interfaces 2017, 9, 38117-38124.

(5) Sigal, G. B.; Mrksich, M.; Whitesides, G. M. Effect of Surface Wettability on the Adsorption of Proteins and Detergents. J. Am. Chem. Soc. 1998, 120, 3464-3473.

(6) Bi, C.; Wang, Q.; Shao, Y.; Yuan, Y.; Xiao, Z.; Huang, J. NonWetting Surface-Driven High-Aspect-Ratio Crystalline Grain Growth for Efficient Hybrid Perovskite Solar Cells. Nat. Commun. 2015, 6, 7747.

(7) Tetsuka, H.; Matsui, T. Non-Ionic Fluorosurfactant Improves Wettability of Nitrogen-Functionalized Graphene Quantum Dots for Integration with Optoelectronic Devices. Chem. Lett. 2018, 47, 850852.

(8) Chappat, M. Some Applications of Emulsions. Colloids Surf., A 1994, 91, 57-77.

(9) Guzey, D.; McClements, D. J. Formation, Stability and Properties of Multilayer Emulsions for Application in the Food Industry. Adv. Colloid Interface Sci. 2006, 128-130, 227-248.

(10) Koroleva, M. Y.; Nagovitsina, T. Y.; Bidanov, D. A.; Gorbachevski, O. S.; Yurtov, E. V. Nano- and Microcapsules as Drug-Delivery Systems. Resour.-Effic. Technol. 2016, 2, 233-239.

(11) Thakur, R. K.; Villette, C.; Aubry, J. M.; Delaplace, G. Dynamic Emulsification and Catastrophic Phase Inversion of Lecithin-Based Emulsions. Colloids Surf., A 2008, 315, 285-293.

(12) Perazzo, A.; Preziosi, V.; Guido, S. Phase Inversion Emulsification: Current Understanding and Applications. Adv. Colloid Interface Sci. 2015, 222, 581-599.

(13) Teh, S.-Y.; Lin, R.; Hung, L.-H.; Lee, A. P. Droplet Microfluidics. Lab Chip 2008, 8, 198-220.

(14) Kelly, B. T.; Baret, J.-C.; Taly, V.; Griffiths, A. D. Miniaturizing Chemistry and Biology in Microdroplets. Chem. Commun. 2007, 1773-1778.
(15) Liu, G.; Wang, X.; Wang, K.; Tostado, C. P.; Luo, G. Effect of Surface Wettability on Internal Velocity Profile during Droplet Formation Process in Microfluidic Devices. Int. J. Multiphase Flow 2016, 80, 188-193.

(16) Wehking, J. D.; Gabany, M.; Chew, L.; Kumar, R. Effects of Viscosity, Interfacial Tension, and Flow Geometry on Droplet Formation in a Microfluidic T-Junction. Microfluid. Nanofluid. 2014, $16,441-453$.

(17) Shui, L.; Pennathur, S.; Eijkel, J. C. T.; van den Berg, A. Multiphase Flow in Lab on Chip Devices: A Real Tool for the Future? Lab Chip 2008, 8, 1010-1014.

(18) Derzsi, L.; Jankowski, P.; Lisowski, W.; Garstecki, P. Hydrophilic Polycarbonate for Generation of Oil in Water Emulsions in Microfluidic Devices. Lab Chip 2011, 11, 1151-1156.

(19) Okushima, S.; Nisisako, T.; Torii, T.; Higuchi, T. Controlled Production of Monodisperse Double Emulsions by Two-Step Droplet Breakup in Microfluidic Devices. Langmuir 2004, 20, 9905-9908.

(20) Wu, N.; Oakeshott, J. G.; Easton, C. J.; Peat, T. S.; Surjadi, R.; Zhu, Y. A Double-Emulsion Microfluidic Platform for in Vitro Green Fluorescent Protein Expression. J. Micromech. Microeng. 2011, 21, 054032 .

(21) Carvalho, R. R.; Pujari, S. P.; Lange, S. C.; Sen, R.; Vrouwe, E. X.; Zuilhof, H. Local Light-Induced Modification of the Inside of Microfluidic Glass Chips. Langmuir 2016, 32, 2389-2398.

(22) Bashir, S.; Bashir, M.; Solvas, X.; Rees, J.; Zimmerman, W. Hydrophilic Surface Modification of PDMS Microchannel for O/W and W/O/W Emulsions. Micromachines 2015, 6, 1445-1458.

(23) Abate, A. R.; Krummel, A. T.; Lee, D.; Marquez, M.; Holtze, C.; Weitz, D. A. Photoreactive Coating for High-Contrast Spatial Patterning of Microfluidic Device Wettability. Lab Chip 2008, 8, 2157-2160.

(24) Nisisako, T.; Okushima, S.; Torii, T. Controlled Formulation of Monodisperse Double Emulsions in a Multiple-Phase Microfluidic System. Soft Matter 2005, 1, 23-27.

(25) Pannacci, N.; Bruus, H.; Bartolo, D.; Etchart, I.; Lockhart, T.; Hennequin, Y.; Willaime, H.; Tabeling, P. Equilibrium and Nonequilibrium States in Microfluidic Double Emulsions. Phys. Rev. Lett. 2008, 101, 164502.

(26) Bhushan, B. Springer Handbook of Nanotechnology; Bhushan, B., Ed.; Springer Berlin Heidelberg: Berlin, Heidelberg, 2004; pp 487536.

(27) McDonald, J. C.; Duffy, D. C.; Anderson, J. R.; Chiu, D. T.; Wu, H.; Schueller, O. J. A.; Whitesides, G. M. Fabrication of Microfluidic Systems in Poly(Dimethylsiloxane). Electrophoresis 2000, $21,27-40$.

(28) Zhou, J.; Ellis, A. V.; Voelcker, N. H. Recent Developments in PDMS Surface Modification for Microfluidic Devices. Electrophoresis 2010, 31, 2-16.

(29) Bauer, W.-A. C.; Fischlechner, M.; Abell, C.; Huck, W. T. S. Hydrophilic PDMS Microchannels for High-Throughput Formation of Oil-in-Water Microdroplets and Water-in-Oil-in-Water Double Emulsions. Lab Chip 2010, 10, 1814-1819.

(30) Fritz, J. L.; Owen, M. J. Hydrophobic Recovery of PlasmaTreated Polydimethylsiloxane. J. Adhes. 1995, 54, 33-45.

(31) Trantidou, T.; Elani, Y.; Parsons, E.; Ces, O. Hydrophilic Surface Modification of PDMS for Droplet Microfluidics Using a Simple, Quick, and Robust Method via PVA Deposition. Microsyst. Nanoeng. 2017, 3, 16091.

(32) Roman, G. T.; Hlaus, T.; Bass, K. J.; Seelhammer, T. G.; Culbertson, C. T. Sol-Gel Modified Poly(Dimethylsiloxane) Microfluidic Devices with High Electroosmotic Mobilities and Hydrophilic Channel Wall Characteristics. Anal. Chem. 2005, 77, 1414-1422.

(33) Abate, A. R.; Lee, D.; Do, T.; Holtze, C.; Weitz, D. A. Glass Coating for PDMS Microfluidic Channels by Sol-gel Methods. Lab Chip 2008, 8, 516-518.

(34) Choi, C.-H.; Lee, H.; Weitz, D. A. Rapid Patterning of PDMS Microfluidic Device Wettability Using Syringe-Vacuum-Induced Segmented Flow in Nonplanar Geometry. ACS Appl. Mater. Interfaces 2018, 10, 3170-3174. 
(35) Hu, S.; Ren, X.; Bachman, M.; Sims, C. E.; Li, G. P. Surface Modification of Poly(dimethylsiloxane) Microfluidic Devices by Ultraviolet Polymer Grafting. Anal. Chem. 2002, 74, 4117-4123.

(36) Schneider, M. H.; Willaime, H.; Tran, Y.; Rezgui, F.; Tabeling, P. Wettability Patterning by UV-Initiated Graft Polymerization of Poly(Acrylic Acid) in Closed Microfluidic Systems of Complex Geometry. Anal. Chem. 2010, 82, 8848-8855.

(37) Wang, Y.; Lai, H.-H.; Bachman, M.; Sims, C. E.; Li, G. P.; Allbritton, N. L. Covalent Micropatterning of Poly(Dimethylsiloxane) by Photografting through a Mask. Anal. Chem. 2005, 77, 7539-7546.

(38) Hu, S.; Ren, X.; Bachman, M.; Sims, C. E.; Li, G. P.; Allbritton, N. L. Surface-Directed, Graft Polymerization within Microfluidic Channels. Anal. Chem. 2004, 76, 1865-1870.

(39) Faure, K.; Blas, M.; Yassine, O.; Delaunay, N.; Crétier, G.; Albert, M.; Rocca, J.-L. Electrochromatography in Poly(Dimethylsiloxane) Microchips Using Organic Monolithic Stationary Phases. Electrophoresis 2007, 28, 1668-1673.

(40) Ebara, M.; Hoffman, J. M.; Hoffman, A. S.; Stayton, P. S. Switchable Surface Traps for Injectable Bead-Based Chromatography in PDMS Microfluidic Channels. Lab Chip 2006, 6, 843-848.

(41) Ma, D.; Chen, H.; Shi, D.; Li, Z.; Wang, J. Preparation and Characterization of Thermo-Responsive PDMS Surfaces Grafted with Poly(N-Isopropylacrylamide) by Benzophenone-Initiated Photopolymerization. J. Colloid Interface Sci. 2009, 332, 85-90.

(42) Sugiura, S.; Imano, W.; Takagi, T.; Sakai, K.; Kanamori, T. Thermoresponsive Protein Adsorption of Poly(N-Isopropylacrylamide)-Modified Streptavidin on Polydimethylsiloxane Microchannel Surfaces. Biosens. Bioelectron. 2009, 24, 1135-1140.

(43) Chen, J.; Huffman, A. S. Polymer-Protein Conjugates. II. Affinity Precipitation Separation of Human Immunogammaglobulin by a Poly(N-Isopropylacrylamide)-Protein A Conjugate. Biomaterials 1990, 11, 631-634.

(44) Heskins, M.; Guillet, J. E. Solution Properties of Poly(NIsopropylacrylamide). J. Macromol. Sci., Part A: Pure Appl.Chem. 1968, 2, 1441-1455.

(45) Cheng, X.; Canavan, H. E.; Stein, M. J.; Hull, J. R.; Kweskin, S. J.; Wagner, M. S.; Somorjai, G. A.; Castner, D. G.; Ratner, B. D. Surface Chemical and Mechanical Properties of Plasma-Polymerized N-Isopropylacrylamide. Langmuir 2005, 21, 7833-7841.

(46) Yakushiji, T.; Sakai, K. Graft Architectural Effects on Thermoresponsive Wettability Changes of Poly(N-Isopropylacrylamide)-Modified Surfaces. Langmuir 1998, 14, 4657-4662.

(47) Du, X.; Cui, H.; Sun, B.; Wang, J.; Zhao, Q.; Xia, K.; Wu, T.; Humayun, M. S. Photothermally Triggered Shape-Adaptable 3D Flexible Electronics. Adv. Mater. Technol. 2017, 2, 1700120.

(48) Wang, J.; Zhao, Q.; Cui, H.; Wang, Y.; Chen, H.; Du, X. Tunable shape memory polymer mold for multiple microarray replications. J. Mater. Chem. A 2018, 6, 24748-24755.

(49) Da Silva, R. M. P.; Mano, J. F.; Reis, R. L. Smart Thermoresponsive Coatings and Surfaces for Tissue Engineering: Switching Cell-Material Boundaries. Trends Biotechnol 2007, 25, 577583.

(50) Patel, N. G.; Zhang, G. Responsive Systems for Cell Sheet Detachment. Organogenesis 2013, 9, 93-100.

(51) Liu, H.; Wang, S. Poly(N-Isopropylacrylamide)-Based ThermoResponsive Surfaces with Controllable Cell Adhesion. Sci. China: Chem. 2014, 57, 552-557.

(52) Xia, Y.; Whitesides, G. M. Soft Lithography. Annu. Rev. Mater. Sci. 1998, 28, 153-184.

(53) Rohr, T.; Ogletree, D. F.; Svec, F.; Fréchet, J. M. J. Surface Functionalization of Thermoplastic Polymers for the Fabrication of Microfluidic Devices by Photoinitiated Grafting. Adv. Funct. Mater. 2003, 13, 264-270.

(54) Yekta-Fard, M.; Ponter, A. B. Factors Affecting the Wettability of Polymer Surfaces. J. Adhes. Sci. Technol. 1992, 6, 253-277.

(55) Shui, L.; Van den Berg, A.; Eijkel, J. C. T. Interfacial Tension Controlled W/O and O/W 2-Phase Flows in Microchannel. Lab Chip 2009, 9, 795-801.
(56) Lee, D.; Weitz, D. A. Double Emulsion-Templated Nanoparticle Colloidosomes with Selective Permeability. Adv. Mater. 2008, 20, 3498-3503.

(57) Shum, H. C.; Lee, D.; Yoon, I.; Kodger, T.; Weitz, D. A. Double Emulsion Templated Monodisperse Phospholipid Vesicles. Langmuir 2008, 24, 7651-7653.

(58) Herranz-Blanco, B.; Arriaga, L. R.; Mäkilä, E.; Correia, A.; Shrestha, N.; Mirza, S.; Weitz, D. A.; Salonen, J.; Hirvonen, J.; Santos, H. A. Microfluidic Assembly of Multistage Porous Silicon-Lipid Vesicles for Controlled Drug Release. Lab Chip 2014, 14, 1083-1086.

(59) Duncanson, W. J.; Lin, T.; Abate, A. R.; Seiffert, S.; Shah, R. K.; Weitz, D. A. Microfluidic Synthesis of Advanced Microparticles for Encapsulation and Controlled Release. Lab Chip 2012, 12, 21352145.

(60) Chu, L.-Y.; Utada, A. S.; Shah, R. K.; Kim, J.-W.; Weitz, D. A. Controllable Monodisperse Multiple Emulsions. Angew. Chem., Int. Ed. 2007, 46, 8970-8974.

(61) Shah, R. K.; Shum, H. C.; Rowat, A. C.; Lee, D.; Agresti, J. J.; Utada, A. S.; Chu, L.-Y.; Kim, J.-W.; Fernandez-Nieves, A.; Martinez, C. J.; Weitz, D. A. Designer Emulsions Using Microfluidics. Mater. Today 2008, 11, 18-27.

(62) Bai, Z.; Wang, B.; Chen, H.; Wang, M. Spatial Wettability Patterning of Glass Microchips for Water-in-Oil-in-Water (W/O/W) Double Emulsion Preparation. Sens. Actuators, B 2015, 215, 330-336.

(63) Abate, A. R.; Weitz, D. A. High-Order Multiple Emulsions Formed in Poly(Dimethylsiloxane) Microfluidics. Small 2009, 5, 2030-2032.

(64) Li, S.; Gong, X.; Mc Nally, C. S.; Zeng, M.; Gaule, T.; AnduixCanto, C.; Kulak, A. N.; Bawazer, L. A.; McPherson, M. J.; Meldrum, F. C. Rapid Preparation of Highly Reliable PDMS Double Emulsion Microfluidic Devices. RSC Adv 2016, 6, 25927-25933.

(65) Lee, H.; Choi, C.-H.; Abbaspourrad, A.; Wesner, C.; Caggioni, M.; Zhu, T.; Weitz, D. A. Encapsulation and Enhanced Retention of Fragrance in Polymer Microcapsules. ACS Appl. Mater. Interfaces 2016, 8, 4007-4013.

(66) Shang, L.; Cheng, Y.; Wang, J.; Yu, Y.; Zhao, Y.; Chen, Y.; Gu, Z. Osmotic Pressure-Triggered Cavitation in Microcapsules. Lab Chip 2016, 16, 251-255.

(67) Ye, B.; Ding, H.; Cheng, Y.; Gu, H.; Zhao, Y.; Xie, Z. Photonic Crystal Microcapsules for Label-Free Multiplex Detection. Adv. Mater. 2014, 26, 3270-3274. 